Aus der k. k. dermatologischen Universitätsklinil [Vorstand: Professor Kreibich] und dem $\mathrm{k}$. $\mathrm{k}$. Institut für mediz. Ohemie [Vorstand: Professor von Zeynek] in Prag.

\title{
Die Jodtherapie in ihren Beziehungen zur quantitativen Jodausscheidung.
}

Von

\author{
Dr. Richard Fischel (Bad Hall).
}

Wenn auch bei Beurteilung der Heilwirkung eines Arzneipräparates die klinische Prüfung ausschlaggebend sein muß, so bieten quantitative Untersuchungen, Verteilung und Ausscheidung der Klinik einen wertvollen Fingerzeig für die Dosierung der Präparate und die Vermeidung von Nebenwirkungen und ermöglichen erst das Verständnis für die richtige Anwendung derselben. Mangelnde Kenntnisse nach dieser Richtung diskreditieren manches Medikament in unverdienter Weise, während das Verständnis der quantitativen Verhältnisse die durch die einführende Reklame hochgespannten Erwartungen auf das richtige Maß reduzieren.

Wenn ich nun von diesem Gesichtspunkte aus die Jodtherapie einer Betrachtung unterziehe, so erhebt sich die Frage, welche Forderungen sind wir berechtigt, an ein klinisch brauchbares Jodpräparat zu stellen? Wir müssen verlangen:

a) daß es wirksam,

b) $\mathrm{daB}$ es, seine pharmakologische Reinheit rorausgesetzt, frei von Nebenwirkungen innerbalb der wirksamen Dosengrenze sei.

Für den ersten Punkt kommt der klinischen Beurteilung eine entscheidende Rolle zu. Als Prüfungsobjekte erweisen Arch. f. Dermat. u. Syph. Bd. XCVII. 
sich gerade die gummösen, dem Gesichte und der tastenden Hand zugänglichen Affektionen und der Kropf als geeignet, während Asthma, Bronchitis, Psoriasis etc. der Phantasie und dem Wohlwollen des Beobachters freiesten Spielraum lassen.

Müssen wir bei der Heilwirkung der Individualität des Patienten einen großen Einfluß zugestehen, so ist der Versuch der Aufstellung von gesetzmäBigen Beziehungen zwischen Krankheitsherd und Heilmitteldose unmöglich.

Herrscht doch bei der Dosierung des Jodkali eine so große Verschiedenheit vor wie fast bei keinem anderen Medikament, so dab Zuelzer mit Recht sagt: "Einzelne verordnen davon $0.5-2$ Gramm pro die, andere geben die fünfbis zwanzigfache Tagesdosis, wieder andere begnügen sich sogar in neuester Zeit mit den im Jodothyrin enthaltenen Milligrammen Jod und verzeichnen gute Erfolge."

In engem Zusammenhange mit der Frage der Dosierung steht die Wahl des Präparates, wobei die Form der Bindung des Jodions und der Prozentgehalt an Jod und der durch die Verschiedenheit der Resorptionsverhältnisse bedingte Applikationsmodus erst die Aufstellung der verschiedenen Indikationen ermöglicht.

Auch bei Vermeidung der Nebenwirkungen müssen die gleichen Momente, Individualität und die von denselben untrennbaren Fragen der Wahl und der Anwendungsweise des Medikamentes zur Diskussion gestellt werden.

Die in Betracht kommenden Präparate sind:

a) die anorganischen, $z u$ welchen die Jodalkalien: JK, $\mathrm{JNa}, \mathrm{JNH}_{4}, \mathrm{JLi}$, dann noch Jodrubidium, Jodstrontium, Jodeisen (Syr. ferri jodati) und die sauerstoffhaltigen Halogene $\left(\mathrm{JKO}_{3}, \mathrm{JNaO}_{3}\right)$ gehören;

b) die organischen Präparate.

Von diesen haben hauptsächlich zwei Gruppen praktischen Verwendung gefunden. Die jodierten Kohlenwasserstoffe, von denen wir die Jodfette (Jodipin, Sajodin) und das Jothion hervorheben wollen und die Jodeiweißpräparate (Jodolen, Jodalbazid, Jodeigon, Jodglidine). 
Das Jodoform ${ }^{1}$ ) und die jodierten Benzolderivate (Sozojodol, Aristol, Europhen) schlossen sich, da sie vorwiegend äußerlich Verwendung gefunden haben, von selbst für die Beantwortung der von mir gestellten Fragen aus.

Wenn ich nun auf die Wirkung der Präparate näher eingehen soll, so sei gleich anfänglich herrorgehoben, daß wir es ausnahmslos mit einer Jodkaliwirkung zu tun haben. In welcher Form immer das Jod im Präparat gebunden ist, die Hauptmenge wird im tierischen Organismus in Jodkali verwandelt.

Ich will es dahingestellt lassen, ob das Jodalkali als solches (Lesser) oder katalytisch (Schade) wirkt ${ }^{2}$ ) oder ob zweitens der Sauerstoff des Blutes bei Gegenwart von $\mathrm{CO}_{2}$ (Kämmerer) das Plasma (Binz), oder drittens der Blutsauerstoff als solcher (Buchheim) nebst seiner Wirkung auf die Gefäßwand die Abspaltung des Jods in den Geweben besorgt, und so dem Jod in statu nascendi der Heilerfolg zuzuschreiben ist.

Um nun die verschiedenen Jodmittel beurteilen zu können, müssen wir die Forderung festsetzen, die wir an das "ideale" Präparat bezüglich der Aufnahme i. e. der Resorption, bezüglich des Verbleibens i. e. der Remanenz und der Ausscheidung des Jods zu stellen berechtigt zu sein glauben.

Ein Jodpräparat soll rasch in ausreichender Menge absorbiert relativ lang im Organismus bleiben (Welander). Sing e r präzisiert seine Ansprüche folgendermaßen: "Die Gesamtmenge des in der ersten Zeit der Joddarreichung ausgeschiedenen Jods darf keinen hohen Wert erreichen.

Die Jodelimination darf in der Zeiteinheit nicht zu hoch sein, sondern soll sich möglichst gleichmäßig während der einzelnen Versuchsperioden hinziehen.

Die Jodelimination soll noch möglichst lang nach der letzten Joddosis in nachweisbaren Mengen dauern."

1) Nach Eingabe von Jodoform werden Jodide und Jodate (organische Jodverbindungen) im Harn ausgeschieden. Die Elimination nach einmaliger Applikation von 5 Gramm kann sich über fünf Wochen ausdehnen (H e fte r).

2) Im Jahre 1842 hat übrigens Scharlau auf die katalytische Wirkung des untersetzten JK aufmerksam gemacht, allerdings auf Grund vollständig unzulänglicher Analysen. 
Ich glaube wohl, daß wir im Gegensatz zu Singer und in Übereinstimmung mit Welander von einem idealen Jodpräparat eine rasche und ausgiebige Aufnahme selbstverständlich nur zur Höhe der wirksamen Dosis erwarten können.

Die möglichst lange gleichmäßige Ausscheidung in der Zeiteinheit und die aus ihr abzuleitende gleichmäßige Verteilung des Jods in den Geweben, bzw. in der Zirkulation und ein rasches Abklingen der Ausscheidung sind die weiteren Kardinalpunkte für das ideale Jodheilmittel.

Bei den folgenden Untersuchungen ist eine normale Tätigkeit des Hauptausscheidungsorgan des Jods, der Niere, unbedingte Voraussetzung, wird es nicht ausdrücklich anders erwähnt.

\section{Ausscheidungsdauer.}

Was nun den Beginn des Auftretens der Jodreaktion im Harn betrifft, so besteht zwischen JK und den organischen Jodverbindungen eine mäßige Zeitdifferenz zu Gunsten des JK, das nach $1 \frac{1}{4}$ Minuten schon im Harn nachgewiesen werden konnte und dessen Erscheinen durchschnittlich 1/4 Stunde nach der Einnahme erwartet werden darf (Literatur, siehe $\mathrm{Heffter}$ ).

Bezüglich des Sajodins konnte ich in zwei Fällen bei einer Dosis von 1 Gramm feststellen, daß es in der ersten Stunde nicht nachweisbar war. Erst in der zweiten Stunde konnte nach Veraschung des Harns ein positiver Befund erhoben werden.

Nach Verabreichung von 1 Gramm Jodglidine war nach 40 Minuten die Chloroformprobe im Harn positiv, nach 20 Minuten negativ.

Ich verzichte auf die detaillierte Wiedergabe des Ausscheidungsbeginns der anderen organischen Jodpräparate, da eine Veraschung des Harns zur richtigen Festsetzung des Termins, wie im Sajodinversuch bewiesen, notwendig ist, diese aber nur in den seltensten Fällen von den mit den einzelnen Präparaten operierenden Autoren vorgenommen wurde.

Was nun die Länge der Ausscheidung betrifft, so läßt sich auch hier ein Gegensatz zwischen JK und den organischen Präparaten nachweisen, wobei zu bedenken ist, ob es in einmaliger Dose oder mehreren über den Tag verteilten 
Dosen rerabreicht wurde. Deutlich sehen wir dies an den Versuchen Antens, der nach 055 Gramm JK 40, nach $z$ wei innerhalb von 5 Stunden genommenen Dosen 56, nach drei innerhalb von 10 Stunden genommenen Dosen 77 stündige Ausscheidung beobachtete. ${ }^{1}$ ) Lifschitz konstatierte nach einmaliger Dosis von 3 Gramm noch am vierten Tage Jodspuren im Harne. Die Abstoßung der im Organismus nach längerer Darreichung angehäuften Jodmengen kann mehrere Wochen mit Unterbrechung dauern (Lifschitz). Für Jodol $(0 \cdot 5)$ hat F. J. Pick gezeigt, dali die Ausscheidungsdauer $4 \frac{1}{2}$ The beträgt, Singer für 10 Gramm Jodipin $41 / 2-5$ Tage. Nach meinen eigenen Untersuchungen nach $3,6,9 \mathrm{Gramm} 25 \%$ Jodipin lassen sich noch nach neun Tagen, allerdings nur nach Verkohlung, Jodspuren im Harne nachweisen. Für Jothion nach Applikation von 2-3 Gramm beträgt die Ausscheidung vier Tage; nach längerer Applikation (durch 17 Tage) konnte Wesenberg noch nach 16 Tagen nach Veraschung ein positives Resultat erzielen. Jodolen. (Sommerfeld) wird nach Darreichung von 2, 4, 6 Gramm in einem Zeitraum ron 3-5 Tagen ausgeschieden. Die Ausscheidungsdauer nach Jodalbazideinnahme beträgt 4-5 Tage, während die gleiche Dosis JK nur 3-4 Tage in Anspruch nabm und für Jodglidine sollen nach Boruttau die gleichen Verhältnisse wie für JK gelten. Auffallend ist es dann, daß nach 6tägiger Darreichung von $3 \times 0.1=0.3$ Gramm Jod pro die die Ausscheidung schon in zwei Tagen beendet war.

Nach meinen eigenen Untersuchungen ist die Ausscheidung des Sajodins nach einer einmaligen Dosis von 1 Gramm in vier Tagen, nach drei über einen Tag verteilten Dosen von 1 Gramm in sechs Tagen beendet.

Aus diesen angefübrten Untersuchungen geht hervor, da $B$ das JK am raschesten eingenommen, aber auch am raschesten ausgeschieden wird, das Jod der organischen Verbindung etwas langsamer in den Kreislauf gelangt, aber auch länger im Organismus verweilt.

1) Jenny verascht nach 40 Stunden $50 \mathrm{~cm}$ Harn, während Anten nur $20 \mathrm{~cm}$ verwendet, womit die Verlängerung der Ausscheidung auf 55 Stunden nach Darreichung von $0.5 \mathrm{JK}$ in den Vers. Jennys ihre Erklärung findet. 
Das Verständnis der Jodverteilung wird man aber durch quantitative Bestimmungen der ausgeschiedenen Mengen wesentlich fördern und so werde ich jetzt das Verhältnis der Jodeinfubr zur Jodausfuhr in toto und in der Zeitheit, soweit mir in der Literatur Daten zugänglich waren und auf Grund eigener Versuchsresultate zu besprechen haben.

\section{Gesamtausscheidung.}

JK, das einen Gehalt von 76.5 Jod besitzt, war schon im Jahre 1842 Gegenstand quantitativer Ausscheidungsversuche, denen zufolge Scharlau zu dem Schlusse kommt, daß es im Organismus genau in derselben Menge ausgeschieden wird, in der es verabreicht wurde.

Antens exakte Untersuchungen ergaben Ausscheidungszahlen von $65 \%, 66 \%, 79 \%$, also im Mittel $70 \%$ der Einfuhr, die $0.5 \mathrm{~g}$. JK betrug. $85 \%$ bei drei über den Tag verteilten Dosen à $0.5 \mathrm{~g} \mathrm{JK}$.

Jenny fand $72 \cdot 28 \%$ nach gleicher Dosis.

Singer nach 1.3 Gramm JK $=1$ Gramm Jod $78 \%$ $(72 \cdot 4,69 \cdot 8,84 \cdot 7 \%)$.

Es wird also zirka $3 / 4$ des eingeführten Jodkali im Harne wiedergefunden. Nach wiederholten Gaben allerdings ein größerer Prozentsatz (Anten).

Interessant sind die Angaben von Lifschitz, die nach einer einmaligen groß̉en Dosis à 3 Gramm $42 \cdot 46 \%$, 43.89, 55.13 (letzteres ein Selbstversuch), 57.63\% Jodkali im Harn wiederfand.

Ein Vergleich dieser Zahlen mit denen Antens, woraus der Schluß folgen würde, das größere einmalige Dosen schlechter ausgenützt werden, ist bei der individuellen Ausscheidungsvariation nicht gestattet, sondern Versuche mit verschiedenen Dosen an demselben Individuum zur Lösung dieser interessanten Frage aufzunehmen.

Kellermann fand das Ausscheidungsverhältnis bei $1 \cdot 5-2.6 \mathrm{JNa}$ pro die bei längerer Darreichung $70 \%, 78 \cdot 4$, $76.3 \%$, allerdings nach einer von Heffter und Wesenberg bemängelten Methode, die das Jodbindungsvermögen des Harns 
Die Jodtherapie in ihren Bezieh. z. quant. Jodausscheidung. 279

ganz unberücksichtigt läBt (Vitali, Marung, Walko, Wesenberg, Schürhof).

Kurz sei auf die Lifs chitzschen Versuche mit größeren Dosen JK hingewiesen, wo nach 20tägiger Einverleibung von 6-12 Gramm - pro die (in Summa 172 Gramm Aufnahme) $143.445=83 \%$, in einem zweiten Fall bei Dosen bis zu 16 Gramm $71 \%$ bei täglich steigernden Dosen bis 15 Gramm $44 \%, 79 \%, 77 \%$ JK der eingeführten Menge zur täglichen Ausscheidung kamen. Hier müssen wir uns vorläufig für Erklärung solcher Ausscheidungsspannungen mit dem Begriffe der "Individualität" begnügen und hinter diesem Ausdruck unsere Unkenntnis verbergen.

Auch äußerlich kann JK in Vaseline und Axungia porci appliziert werden (Capellmann, Xylander). Es kommen zirka $20 \%$ des JK zur Ausfuhr durch den Harn. Doch kommt diesem Verfahren wegen der Hautreizung (Hirschfeld und Pollio, Lion) und der ungenügenden Resorption nur eine untergeordnete Bedeutung zu.

Von Jodolen einem Jodol-Eiweißpräparat mit $36 \%$ Jod wird von 0.4 Gramm $0.316=79 \%$, von $0.3,0.27=66 \%$, von $0.455,0.191=43 \%$ ausgeschieden.

Wir finden also sehr bedeutende Schwankungen, bei denen die höchste Prozentzahl nahezu das Doppelte der niedrigsten beträgt.

Nach Boruttau kommen von 3.0 Gramm Joglidine $=0.3 \mathrm{Jod}=68.1 \%{ }^{1}$ ) für den Menschen, von 4 Gramm $=0.4$ Jod $=63.4 \%$ Jod für den Hund im Harn wieder zum Vorschein.

Bei sechs Tage langer Darreichung von $3 \times 0.3$ Jodglidine wird $59 \cdot 2,61 \cdot 7$, bei täglich $3 \times 0.15 \mathrm{Jodglidine} 95 \%$ ausgeschieden. Auffallend ist es, wie schon früher erwähnt, daß innerhalb zweier Tage die Ausscheidung beendet ist, was bei der Ähnlichkeit mit den Lifs ch üt $\mathrm{z}$ schen Versuchen eigentlich nicht erwartet werden sollte.

Über das von Volkmar Klopfer in Leipzig erzeugte Jodglidine teilt Boruttau mit, dall es eine Jodverbindung

1) Nach den von Boruttau mitgeteilten Zahlen, der das Ausscheidungsverhältnis mit $65 \%$ angibt, ergäbe sich als Resultat $68 \cdot 1 \%$, worauf später noch näher eingegangen werden soll. 
des Pflanzeneiweibesglidine sei, aus welcher durch die Pepsinverdauung, Behandlung mit Alkohol oder kurz dauerndes Kochen mit verdünnter Säure kein freies Jod abgespalten wird. Es wird durch eine von allen bisherigen verschiedene Jodierungsmethode gewonnen, bei welcher keinerlei Zersetzung des nativen Eiweißes stattfindet, trotzdem die Jodierung eine feste ist.

Meine Untersuchungen ergaben, daß nach Zusatz von zirka $1 \mathrm{~cm}^{3}=0.05 \%$ HCL nach fünf Minuten im Brutschrank bei $37^{\circ} \mathrm{C}$. bereits reichlich freies Jod nachzuweisen war.

Über das Jodalbazid (7-8\% Jodgehalt) und Jodeigon konnte ich quantitative Ausscheidungsresultate nicht finden.

Dem Jodglidine kommt im Gegensatz zu diesen zwei Präparaten nach Feigl eine viel leichtere Abspaltbarkeit des Jods $\mathrm{zu}$, in dem nur ein geringer Teil des Jods festgebunden ist, der Rest als beigemengt oder in starrer Lösung vorhanden gedacht werden muß, während bei Jodalbazid und Jodeigon das Jod fest in das Protein eingelagert sein soll.

Dem entspricht auch die sekretionsfördernde Wirkung auf die Magensaftabsonderung, die von den mit freiem Jod erzielten Resultate nur wenig abweicht.

Das Jothion, das ja nur äußerlich zur Anwendung gelangt, da ihm nach Einnahme per os narkotische Eigenschaften zukommen (Dreser), enthält $71 \cdot 74$, bzw. $72 \cdot 06 \%$ Jod. In vier ${ }^{1}$ ) Versuchen von Wesenberg (Einreiben von 2-3 Gramm) auf die Brust wurde im Versuch I und III $25.8 \mathrm{bzw} .32 .3=29.1 \%$ im Mittel, im Versuch II und IV 13.9\%, 15.1\% $=14.5 \% \mathrm{im}$ Mittel Jod ausgeschieden. Nach Kellermann kommt aus einer Jothion-Lanolinsalbe $\left(\begin{array}{lll}\overline{\text { aa }} & 6.0 & \mathrm{~g}\end{array}\right) \quad 10 \%$, von $6 \mathrm{~g}$ der gleichen Salbe $5 \%$ und einem Jothion 12.5 Glyzerin Spiritus aa $6.25 \mathrm{~g}$ Gemisch $18 \% \mathrm{~J}$ zur Ausscheidung. Wesenberg fand (nach seiner letzten Mitteilung) nach 20\%-Jothion-Vasogene-Applikation zirka $26-28 \%$ Jod im Harne wieder. Ich gebe die Resultate von zwei eigenen Versuchen, die zu anderem Zwecke von mir ausgeführt wurden, wieder.

Die angewandte Methode der quantitativen Jodbestimmung war für größere Jodmengen die von Fresenius-Blum. Für kleinere Jodmengen das von Anten modifizierte Rabourdin sche Verfahren.

1) Selbstversuch und Laboratoriumsdiener. 
17jähriges Mädchen. Lupus des unteren Augenlides und des linken Oberarmes. Am 9. Januar 1909 auf Brust und Rücken auf ein zirka $6 \mathrm{~cm}$ breites und $20 \mathrm{~cm}$ langes Rechteck der Haut durch zirka drei Minuten $2 g$ Jothion eingepinselt. Also zu $70 \%$ Jod angenommen $=1.4 \mathrm{~g}$ Jod. Ausgeschieden $0 \cdot 26748=19 \cdot 71 \%$.

St., 21jährige Patjentin. Lues gummosa. Zwei handtellergroße Brustfläche mit $2 g$ Jothion eingepinselt.

Ausgeschieden am 1. Tag 0.4554,

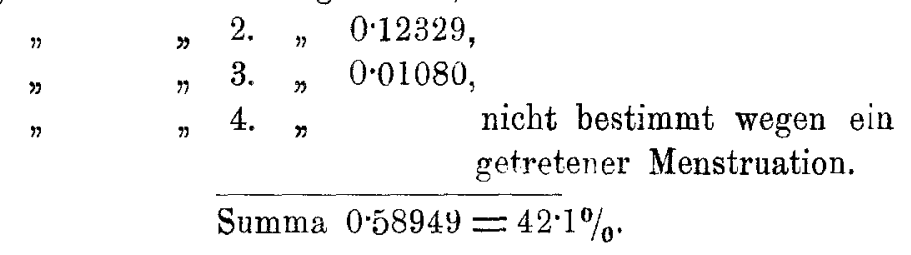

Der Prozentgehalt ist natürlich noch um ein Geringes größer, da der Harn des 4. Tages vernachlässigt wurde.

ob hier der hohe Perzentgehalt auf die Menstruation zurück zuführen ist, lasse ich dahingestellt. (Hyperämie der Mammae und der über denselben befindlichen Hautpartien?) Fall 1 befand sich ebenfalls knapp vor der Menstruation, doch war nur die Hälfte des Jothions auf die Brust, die andere Hälfte auf den Rücken gepinselt worden.

Von in vier einander folgenden Tagen à $2 \cdot 4 \mathrm{~g}$ eingeriebenem Jothion wurden in Wesenbergs Versuch in 5 Tagen $30.88 \%$ Jod ausgeschieden. Fine wesentliche Steigerung der im Harne gefundenen Jodmengen ist also bei fortgesetzter Jothiondarreichung nicht erzielt worden.

Begüglich des 10\%igen Jodipins fand Singer: daß nach einmaliger Aufnahme von $10 \mathrm{~g}=1 \mathrm{~g}$ Jod $49 \cdot 2-69 \cdot 2 \%=58.5 \%$ Jod als Mittel im Harn erschienen. Größere Schwankungen also als bei JK, bei dessen Darreichung 69.8 bis $84 \cdot 7$, demnach eine Ausscheidungsbreite von $15 \%$ vom gleichen Autor nachgewiesen wurde. Doch werden wir diese Angabe vor der Verallgemeinerung mit Vorsicht aufnehmen, wenn wir uns an die oben zitierten Resultate der Lifschitzschen Versuche nach Darreichung von $3 g$ JK erinnern. Nach 3 mal $2.5 g$ Jodipin $=$ 
$2.25 \mathrm{~g}$ Jod erscheint $1.48 \mathrm{~g}$ im Harn $=66 \%$ gegen $58 \%$ nach einmaliger größerer Gabe, so daß bei dieser die Bedingung für den Ansatz von Jodfett nach Singer günstiger sein sollen.

Bedenken wir aber, daß unter den 8 Fällen mit einmaliger innerlicher Jodipindosis auch Fälle von $63 \%$ (bzw. 69\% im Hungerversuche an dem gleichen Patienten) und $69 \%$ Jodausscheidung sich befanden, so werden wir der Angabe über erhöhte Ausscheidung nach mehrfachen kleineren Dosen eine absolut beweisende Kraft nicht zuerkennen können. Wintern itz fand nach $53 \mathrm{~g}$ innerhalb 4 Tage gereichten Jodipins $4.3 \mathrm{~g} \mathrm{Jod}$ durch den Harn ausgeschieden, also $61.8 \%$. 0.18 Jod waren durch den Kot unausgenützt verloren gegangen.

Bezüglich der Jodipininjektion am Menschen existieren genaue Angaben über das gesamte Jodausscheidungsquantum nicht, da äußere Gründe die Bestimmung der sich über Monate erstreckenden Ausfuhr sehr erschweren.

Das jetzt gebräuchlichste Ersatzmittel des JK, das Sajodin (Bayer in Elberfeld, Meister Lucius und Brüning-Höchst), wurde von mir einer Untersuchung unterzogen. Es ist das Kalziumsalz der Monojodbehensäure, die aus der Erucasäure des Rüböls durch Anlagerung ron Jod wasserstoff entsteht $\left(\mathrm{C}_{22} \mathrm{H}_{49} \mathrm{O}_{2} \mathrm{~J}\right) \mathrm{Ca}_{42}$. Es enthält $26 \%$ Jod, $4 \cdot 1 \% \mathrm{Ca}$ (Fischer und Mering).

Zernik schreibt: "Das viel angewendete und aus fabrikstechnischen Gründen und um den ohnehin nicht billigen Preis des Mittels nicht noch mehr zu erhöhen, in nicht absolut chemisch reinen Zustand in den Handel gebrachte Präparat enthält nicht $26 \%$ Jod wie dem chemisch reinen monojodbehensauren Kalzium entsprechen würde, sondern nur 24.5 , berechnet auf das wasserfreie Präparat. Da aber das Sajodin des Handels fast $1 \cdot 5-2 \%$ Feuchtigkeit enthälî, so beträgt der Jodgehalt des Sajodins, das in Apotheken deponiert wird, noch etwas weniger."

Ich habe daher das Sajodin zu $24 \%$ Jodgehalt angenommen, und diese Zahl meinen Untersuchungen zugrunde gelegt.

$\mathrm{Abderhalden}$ und $\mathrm{Kautz} \mathrm{sh}$ haben in einem Versuch an einem Hund $\left.{ }^{1}\right) 5.8 \mathrm{~g}$ Sajodin $=1.392 \mathrm{~g}$ Jod verfüttert und $0.727=52.227 \%$ Jod im Harne wieder gefunden.

$\left.{ }^{1}\right)$ Gewicht leider nicht angegeben. Jedenfalls handelt es sich in Relation zum Menschen um eine verhältnismäßig große Gabe. 
Ich habe bei einem gesunden kräftigen jungen Menschen (Fall I) nach $1.0 \mathrm{~g}$ Sajodin $=0.24 \mathrm{Jod}$ entsprechend $0.314 \mathrm{JK}$, $0.0072 \mathrm{~J}$ entsprechend $0.0942 \mathrm{JK}$, also $30 \%$ des eingeführten Jods im Harne wieder gefunden. Im Falle II wurde nach $1 \mathrm{~g}$ Sajodin nur durch 2 Tage die Jodausscheidung in mehrstündigen Intervallen bestimmt. Der Einfuhr von 0.24 Jod entsprach eine Ausfuhr von 0.07022 Jod. Bedenkt man, daß in den letzten 2 Tagen im Versuch I nur $4 \mathrm{mg}$ ausgeschieden wurden, so ist die Übereinstimmung eine ausgezeichnete. Es wurden auch von dieser Versuchsperson zirka $30 \%$ der eingeführten Jodmenge im Harne entleert. Nach $3 g$ Sajodin, die in 3 Dosen à $1 g$ über den Tag (Versuch 3) verteilt wurden, ersieht man:

Von $3 \mathrm{mal} 1 \mathrm{~g}$ Sajodin $=0.72 \mathrm{~J}=0.942 \mathrm{JK}$ ausgeschieden $0.3166 \mathrm{~J}=0.414 \mathrm{JK}=43.9 \%$. Es ergibt sich also eine Übereinstimnung mit Anten, der nach mehrfachen Tagesdosen eine prozentisch erhöhte Jodelimination fand. Ob dies die Regel ist, oder hier nicht individuelle Verhältnisse mitspielen, muß durch Versuche an ein und demselben Individuum entschieden werden.

Da Sajodin einen so kleinen Jodgehalt besitzt, $24 \%$ gegen $76.5 \mathrm{JK}$ und $84.6 \% \mathrm{JNa}$ und die Ausscheidungsquote nur $30 \%$ bzw. $44 \%$ beträgt gegenüber $70 \%$ bzw. $85 \%$ (Anten) bei JK, die Wirksamkeit des Präparates durch ernste klinische Publikationen außer Zweifel steht (Roscher), so muß man sich die Frage vorlegen, ob nicht das Recht auf Seite derjenigen ist, die unsere bisherigen Joddosen für viel zu groß ansehen und mit kleineren Dosen ihr Auskommen finden und ob bei den organischen Jodpräparaten (Sajodin) nicht Faktoren maßgebend sind, die die kleinen Dosen wirksam gestalten.

\section{Jodausscheidung in der Zeiteinheit.}

Nach Erörterung des Verbältnisses zwischen Jodeinfuhr und Jodausscheidung ist die Orientierung ïber die Frage der auf die Zeiteinheit berechneten Jodausscheidung geboten. Nach Aufnahme einer einzigen Dosis $0.5 \mathrm{~g} \mathrm{JK}$ auf nüchternem Magen um $7 \mathrm{Uhr}$ früh in $50 \mathrm{~cm}$ Wasser gelöst, fand Anten im ersten Versuch die höchsten Werte der Jodausscheidung in der dritten, im zweiten Versuche in der ersten, im dritten Versuche in der zweiten Stunde. 
In späteren Selbstversuchen hat Anten immer die zweite Stunde als Maximum erkannt, ebenso in einem zweiten nicht ausfübrlich publizierten Versuche bei einer anderen Person; von da ab Verminderung der stündlich ausgeschiedenen Jodmenge, mit neuerlichem Anstieg derselben ron 2-3 nachmittags und 8 bis 9 Uhr abends. Während der ersten 12 Stunden hatte zirka die Hälfte des eingeführten Salzes den Organismus verlassen.

Studeni und Jenny berichten über einen viel unregelmäßigeren Verlauf der stündlichen Ausscheidungskurve. Bei Jenny wurde 2mal das Maximum in der 6. Stunde erreicht.

Ich werde auf diese Schwankungen bei Besprechung meiner eigenen Sajodinversuche noch zurückkommen.

Dem JK zunächst steht Jodglidine. Die Zahlen, die Boruttau über die in den einzelnen Intervallen zu Tage getretenen Jodmengen gefunden hat, sind folgende:

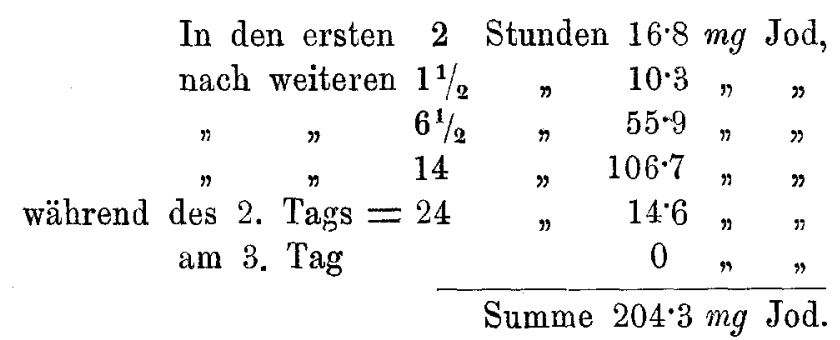

(Bor uttaus Summe : $0.1982=$ Boruttaus \% Zahl $=65 \%$.)

Es muß hier bemerkt werden, daß sich ein mir unerklärlicher Rechenfehler eingeschlichen hat. Weder die Summe noch die Prozentzahl des ausgeschiedenen Jods stimmen. Denn angenommen die von B o rutta u angeführte Summe 0.1982 $g$ ist richtig, so muß die Prozentzahl 66.08 lauten. Für die von mir ausgerechnete Summe 204.3 $\mathrm{mg}$ Jod aber : $68 \cdot 1 \%$.

B or u t t a u schreibt nun: In Hinsicht auf die Dauer der Ausscheidung, ebenso wie auf den Prozentsatz des hier ausschlie日lich in Form von Jodkali ausgeschiedenen Jods war das Verhalten das gleiche wie bei der Einfuhr von Jodalkali.

Die Behauptung Boruttaus, daß das Maximum nicht gleich nach Einführung stattfindet, sondern erst in den zweiten 12 Stunden, läßt sich aus seiner Angabe nicht erweisen. 
Die Jodtherapie in ihren Bezieh. z. quant. Jodausscheidung. 285

In den ersten 2 Stunden $16.8 \mathrm{mg}$, pro Stunde $8.4 \mathrm{mg}$ Jod.

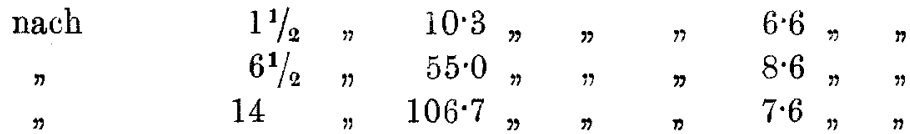

Ich bin mir wohl bewubt, dab die proportionale Berechnung nicht gerechtfertigt ist, doch ist durch die Größe des entscheidenden Zeitintervalls, $61 / 2$ Stunden, in der bei Umrechnung auf stündliche Ausscheidung die größte Menge von Jod ausgeschieden wird, die Feststellung des Maximums verschleiert.

Die Behauptung Boruttaus, daß das Maximum in die zweiten 12 Stunden fällt, ist jedoch durch nichts begründet, da die absolute Größe der Zahl 106.7 doch nicht entscheidet, sondern nur die relative Ausscheidung in der Zeiteinheit (also pro Stunde) mit der hier gebotenen Reserve herangezogen werden könnte.

Neue Versuche sind, falls das Präparat sich klinisch einbürgern sollte, daher unbedingt notwendig.

Die Ausscheidungsdaten des Jodolens (Rösel) sind nicht auf die Zeiteinheit berechnet, so daß ein genauer Finblick in die Ausscheidungsverbältnisse nicht möglich ist.

Die Untersuchung Wesenbergs, der mit Zeitintervallen von $2^{1} / 2$ Stunden angefangen operierte, ergibt für Jothion: das Maximum fälit im Versuche I zwischen die 6.-10. Stunde, Versuch II in die 5.-8. Stunde, Versuch III in die 3.-6. Stunde. Jedenfalls geht aus diesen Zahlen hervor, daß es weiter hinausgeschoben ist, als bei JK, was sich aus der langsam verlaufenden Hautresorption ungezwungen erklärt. Innerhalb der ersten 12 Stunden wurden nur im Versuche I $8.4 \%$ der eingeführten Menge, nach 24 Stunden $15.9 \%{ }^{1}$ ) ausgeschieden.

1) Hier ist Wesenberg ein Versehen widerfahren. Er schreibt: "Nach 24 Stunden sind nach Anten $59 \cdot 3,61 \cdot 3$ und $69.1 \%$ der gesamten Jodmenge des Harns ausgeschieden. In meinen Versuchen hat Versuch I entsprechend nach $221 / 2$ Stunden $61.7 \%$ etc. der gesamten Harnjodmenge von sich gegeben." Bei Wesenberg handelt es sich um das Verbältnis zwischen Jodmenge der Harneinzelportion zur gesamten Harnjodmenge, bei Anten um das Verhältnis der ausgeschiedenen Harnjodmenge zur eingeführten Menge. Zahlen, die sich also mit einander nicht vergleichen lassen und im obigen Sinne richtig gestellt werden müssen. 
Um über die Gleichmäbigkeit der Jodausscheidung und der aus derselben resultierenden Schluß der Jodzirkulation im Organismus ein Urteil zu fällen, ist es notwendig, das ausgeschiedene Quantum in der Einzelportion mit der Tage:menge zu vergleichen.

Während also in den Antenschen Versuchen nach 24 Stunden $91 \cdot 3,92 \cdot 3$ und $86.9 \%$ Jod ausgeschieden sind, sind in den drei Versuchen von W esenberg nach 24 Stunden $63 \cdot 5$, nach 20 Stunden $63 \cdot 5$, nach $221 / 2$ Stunden $61 \cdot 7 \%$ ausgeschieden. Diese so umgerechneten Zablen ergeben deutlich, daß beim JK innerhalb 24 Stunden die Ausscheidung fast ganz beendet ist, von Jothion aber noch nicht ganz $2 / 3$ der ausgeschiedenen Menge des gesamten Quantums im Harne erscheint. Sind im ersten Versuch Wesenbergs in $22 \%$ Stunden $61 \cdot 70 \%$ Jod ausgeschieden worden, so steigert sich die Zahl am 2. Tag bis 86.98 , am 3. bis zu 96.21 und am 4. Tag bis zu $100 \%$. Es wird also am 2. Tag 25, am 3. Tag 10 und am 4. Tag $4 \%$ Jod ausgeschieden.

Langsamerer Anstieg zum Maximum, gleichmäßigere Verteilung (siehe die Kurren Wesenbergs) über einen größeren Zeitraum sind die Vorteile des Präparats gegenüber JK. Die geringe Resorption (über die Depotbildung bei Jothiondarreichung bestehen noch keine Versuche) ist der Nachteil des Mittels.

Das Studium der Jodausscheidungsverhältnisse bei innerlicher Darreichung des Jodipins ergibt nach Sing er als Mittel von an 6 Fällen mit $1.3 \mathrm{~g} \mathrm{JK}$ bzw. $10 \mathrm{~g} 10 \%$ Jodipins angestellten Versuchen folgende Resultate:

A. Für Jodkali. Es werden ausgeschieden (absolute Menge in $m g$ JK bezogen auf die Gesamtausscheidung):

nach $6 \mathrm{St}$., nach weiteren $6 \mathrm{St}$. nach weit. $12 \mathrm{St}$., nach weit. $24 \mathrm{St}$.

$\begin{array}{lccc}480 & 170 & 100 & 32 \mathrm{mg} \mathrm{JK} \\ 61 \% & 22 \% & 13 \% & 4 \% \\ & (83 \%) & (96 \%) & \end{array}$

B. Für Jodipin gilt :

nach $6 \mathrm{St}$., nach weiteren $6 \mathrm{St}$., nach weit. $12 \mathrm{St}$., nach weit. $24 \mathrm{St}$.

$\begin{array}{cccc}164 & 177 & 162 & 66 \mathrm{mg} \mathrm{JK} \\ 28 \% & 30 \% & 29 \% & \left.11 \%{ }^{1}\right) \\ & (58 \%) & (87 \%) & \end{array}$

1) Für die folgenden 3 Tage (die Gesamtausscheidung des Jods dauerte doch 4 Tage) bleiben nach Singer noch $2 \%$ der Gesamtausscheidung übrig. 
Es treten also nach Jodkalidarreichung in den ersten 6, 12, bzw. 24 Stunden 61, bzw. 83, bzw. 96\% der Gesamtausscheidung zu Tage, während bei Jodipin die langsame Resorption durch einen langsamen Anstieg sich verrät. Aber auch in der Zeiteinheit ist eine gleichmäßigere Durchströmung des Organismus mit Jodalkali gewährleistet und der Überflutung der Gewebe, wie sie bei JK Aufnahme stattfindet, bis zu einem gewissen Grade vorgebeugt.

Die bisherigen Angaben der Jodquanten im Harn nach Jodipininjektionstherapie von $F$ eibes, meines Wissens die einzigen nach dieser Richtung hin am Menschen angestellten Versuche, sind ohwe Einschränkung in der Literatur hingenommen wurden. Feibes nimmt von einer möglichst frisch gelassenen Harnportion 25 bis $100 \mathrm{~cm}$ zur Verarbeitung und rechnet dann die gefundenen Jodmengen auf einen Liter Harn um. Ob der Harn stets zur gleichen Tagesstunde entleert wurde, wird nicht mitgeteilt. Doch hätten Feibes seine eigenen Analysen belehren müssen, daß eine einfache Multiplikation der Jodmenge mit 40, bzw. 10 zur Feststellung des Literquantums rom wissenschaftlichen Standpunkte aus nicht statthaft war. So fand Feibes im Fall S. morgens pro Liter 0.12888, abends $0 \cdot 20048$, also pro Liter eine Differenz von 0.07160 , abends nahezu das Doppelte des Morgenwertes. Am folgenden Tag 0.18974 am Morgen, am Abend 0.24344. Die Differenz beträgt pro Liter 0.5370, also auch hier noch $25 \%$. Viel besser, aber noch nicht genügend wäre es gewesen, wenn $\mathrm{F}$ eibes wenigstens die Größe der Tagesportion, auf der seine Untersuchung basiert, angegeben hätte, um so wenigstens reelle Werte mitzuteilen. Bei dem großen Interesse, das diese Frage schon in physiologischer Beziehung bietet, bedarf es einer nochmaligen Bearbeitung des Gegenstandes mit Berücksichtigung des reellen Tagesquantums.

Dieser kritischen Notiz eingedenk lassen sich die Feib esschen Resultate folgendermaßen zusammenfassen: Im allgemeinen erschienen in den, den ersten Jodipininjektionen folgenden Tagen kleine Mengen Jod im Harne (erst nach Behandlung des Urins mit kochender Kalilauge nachweisbar). Die Elimination steigt dann langsam und ganz regelmäßig an. Über 
einen gewissen Betrag scheint die Ausscheidungsmenge des Jods nicht hinauszugehen, ob nun 25, 35 oder sogar 50 Injektionen à $20 \mathrm{~cm} \mathrm{25 \%}$ Jodipin gemacht wurden. Vom 10. bis 15. Tag ab ist erst ein Dekagramm Jod im Liter Trin nachzuweisen. Die größte Jodmenge pro Liter Urin betrug 0.48185 am Tage nach der 40. Injektion. (Fall H.) Obwohl noch $11 \mathrm{In-}$ jektionen gemacht wurden, sank der Jodgehalt des Urins wieder. Durchschnittlich werden $0.2 \%$ Jod pro Liter Urin ausgeschieden. Das Abklingen der Ausscheidung zieht sich ebenfalls über einen langen Termin hin und so wurden noch nach 219 Tagen 0.08144 $g$ Jod im Liter Harn ausgeschieden. Selbstverständlich verlieren die an die balneotherapeutischen Versuche geknüpften Schlußfolgerungen von F eibes auf Grund obiger Bemerkungen den ihnen zugesprochenen Wert.

Wichtig fuir die Beurteilung der Frage ist der exakten Durchführung wegen ein Versuch von Winternitz an einem Hund (50 Pfund), der an 5 aufeinander folgenden Tagen $161 \mathrm{~g}$ $25 \%$ Jodipin subkutan bei reichlicher Nahrung erhielt.

I. 5tägige Injektionsperiode $7400 \mathrm{~cm} 0.285 \mathrm{~g} \mathrm{JK}$ pro die $0.057 \mathrm{~g} \mathrm{JK}$

II. 5 "der Injektion fol. Per. 7309 " 0.848 " " " 0170 " "

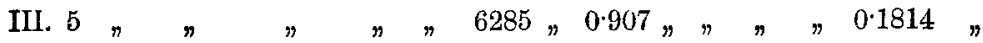

IV. Für 63-68 Tage n. d. Inj. 11050 "0.872, " " " 0.174 " "

V. "68-73" " " " 9465 "0.875" " " 0.175 "

Überraschend ist die Gleichmäßigkeit der Jodausscheidung. 5 Tage nach der letzten Injektion beträgt die an einem Tage ausgeschiedene Jodmenge fast eben so viel als 2 Monate später. Nach 210 Tagen, 7 Monate später, sind in 2 Liter Harn noch $0.0195 \mathrm{~g}$ bestimmt worden. Also sehr langsamer Anstieg, ungleich langsamer als bei den bisherigen Beobachtungen, daneben gleichmäßige Ausscheidung bei längerer Remanenz und langsames Abklingen.

Die rektale Anwendung des Jodipins ist irrationell. Es findet zwar, wie nicht anders zu erwarten, eine Resorption statt, aber sie geht über $10 \%$ nicht hinaus (Winternitz). Durch Zusatz von Pankreassubstanz kann die Ausbeute erheblich gesteigert werden, doch kommen auch hier individuell große Schwankungen vor. 
Über die Resorption ron der Einspritzungsstelle aus verdanken wir Peters genaue, durch Zahlen belegte Angaben, denen zufolge an zwei Versuchstieren nach 8 Tagen an der Injektionsstelle die gleiche Menge Jodipin wie zur Zeit der Einspritzung gefunden wurde, bei den anderen Tiere eine langsame Jodabspaltung nachgewiesen wurde.

Schreite ich nun zum Bericht über die von mir ausgeführten Jodanalysen nach Sajodingebrauch, so ergibt sich:

Fall I. F., 20jähriger Student, nimmt um 8 Uhr früh $1 \mathrm{~g}$. Sajodin entsprechend $0.314 \mathrm{~g} \mathrm{JK}=0.24 \mathrm{Jod}$.

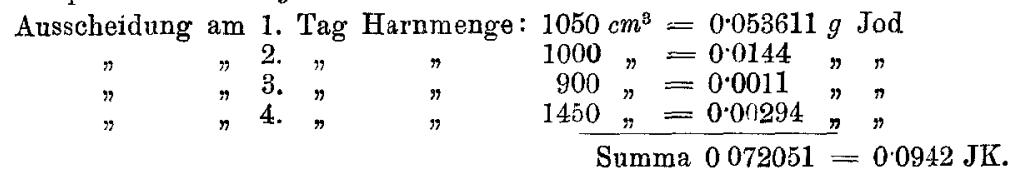

Es werden also am ersten Tag $74 \cdot 4 \%$ der gesamten Jodausscheidung im Harne wieder gefunden. Im 2. Versuch wurde in den 24 Stunden nach Aufnahme von $1 \mathrm{~g}$ Sajodin 0.0554188 $g$ Jod ausgeschieden, Zahlen, welche mit dem ersten Versuch übereinstimmen.

Also auch hier verlassen innerhalb der ersten 24 Stunden zirka 74\% der gesamten Ausscheidung durch den Harn den Körper. Ziehen wir aun JK zum Vergleich heran, so ergibt sich, daß nach Singer $96 \%$ und nach Anten $90 \%$ im Durchschnitt, nach Applikation von Jothion $86.98 \%$ bereits innerhalb des 1. Tages den Organismus verlassen, so daß Sajodin mit $74 \%$ wohl die günstigsten Verhältnisse bezüglich der proportionalen Ausscheidung darbietet.

Was die Verteilung der Jodausscheidung über den Tag betrifft, so lehrt dies die umstehende Tabelle.

Bezüglich der Gesamtausscheidung in diesem Versuche sei nochmals darauf hingewiesen, daß es sich eigentlich um ein künstlich konstruiertes Gesamtquantum handelt, da die Ausscheidung der letzten zwei Tage nicht untersucht wurde. Indem diese bei dem ersten nahezu die gleichen Resultate gebenden Versuche nur $5 \%$ der Gesamtausscheidung beträgt, so kann der Fehler des künstlich konstruierten Gesamtquantums gewiß nur einen sehr geringen Prozentsatz betragen. 


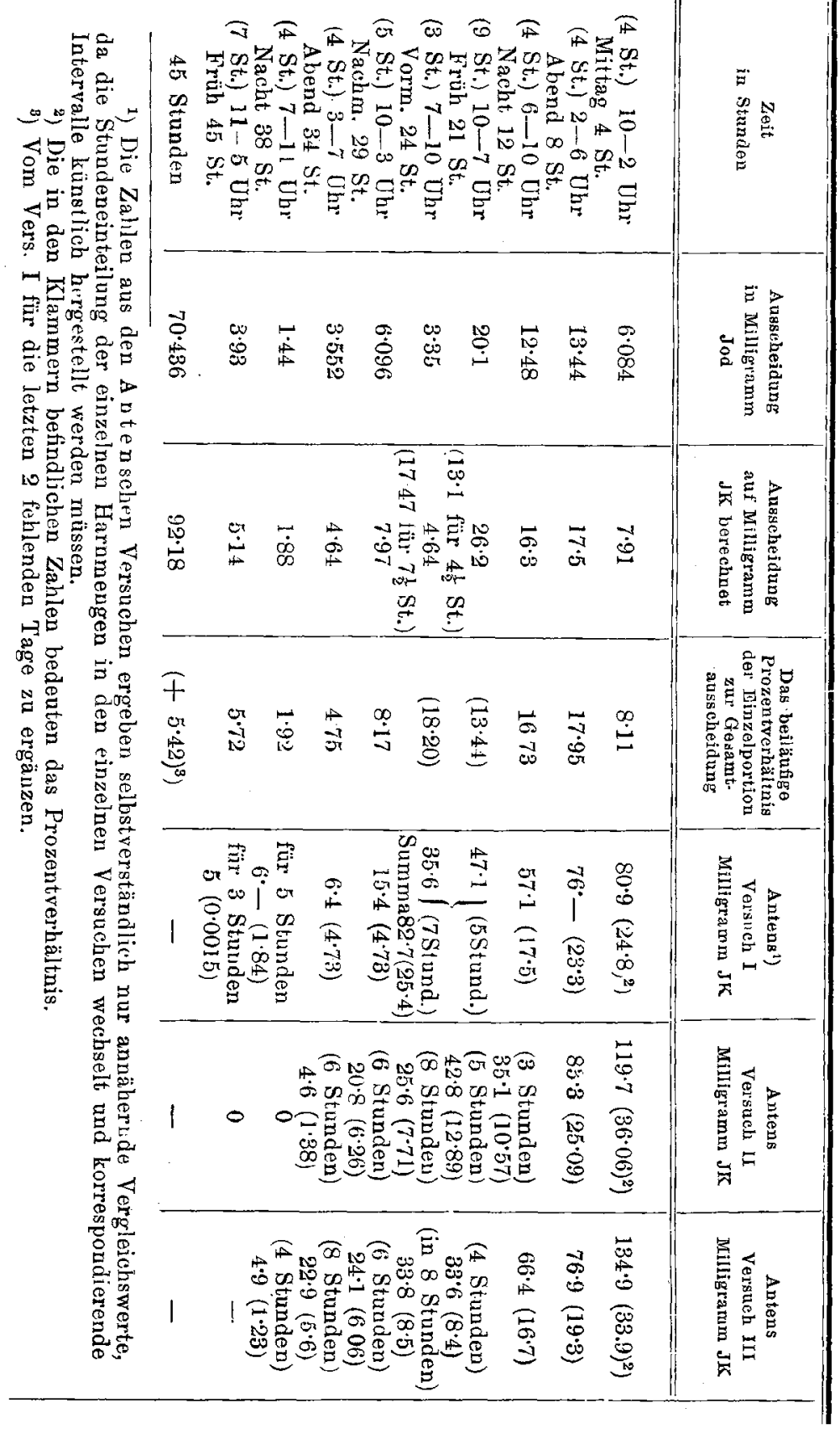


Die Jodtherapie in ihren Bezieh. z. quant. Jodausscheidurg. 291

Ein Vergleich mit den Antenschen JK Zahlen ergibt für das Sajodin gleichmäßigeren langsameren Anstieg und gleichmäßiges langsames Abklingen der Jodausscheidung.

Es sei hier noch ein Versuch erwähnt, der die stündliche Jodausscheidung nach $1.5 \mathrm{~g}$ Sajodin zum Zwecke hatte. Leider wurde das Medikament durch ein Versehen der Wärterin nicht in Oblaten, sondern in Pulverform in Wasser suspendiert gereicht, so daß durch die Adhäsion des Pulvers ein Teil am Glase haften blieb. Da mir für die Wiederholung des Versuches keine Zeit bleibt, so sei er mitgeteilt. Man ersieht die stündlichen Schwankungen der Ausscheidung, ihre proportionalen Verhältnisse, wenn auch die Beziehungen zur Gesamtausscheidung einer Berechnung entgehen. Während des zwölfstündigen Versuches ließ sich im Harne durch die Salpetrigesäure-Chloroformprobe kein Jod nachweisen, was sich auch leicht erklären läßt, wenn man folgende Zahlen berücksicltigt:

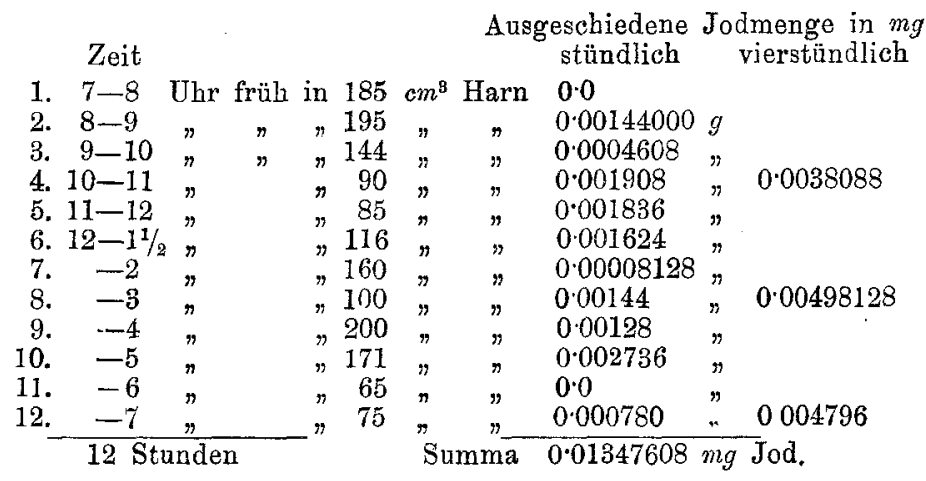

Auch in diesem Versuch zeigt sich eine gewisse Übereinstimmung mit dem Vorhergehenden, in welchem die Harnmenge in größeren Intervallen der Jodanalyse unterzogen wurde.

Die Jodmenge steigt in den zweiten 4 Stunden beträchtlich im Vergleich zum ersten Zeitabschnitt, in den 3. folgt ein leichtes Absinken, also eine Übereinstimmung mit dem vorhergehenden Versuch II. Was nun die Verteilung auf die einzelnen Stunden betrifft, so wird das'Maximum erst in der 11. Stunde erreicht, im scharfen Gegensatz zum JK, das meist schon in der zweiten Stunde die höchsten Ausscheidungswerte ergibt. In der ersten 
Stunde ließ sich kein Jod nachweisen, dann wird zwischen 10-12 ein Gipfel erreicht. Ein Absinken erfolgt bis 3 Uhr nachmittag, dann wieder ein Maximum um 5 Uhr. Sollten sich diese Erhebungen in den Nachuntersuchungen als konstant erweisen, so können sie mit den von Witt nach Jodkalidarreichung beobachteten Schwankungen, die mit den ron $\mathrm{Rose}$ $\mathrm{mann}$ für die Stickstoffausscheidung, von Röske für die Phosphorausscheidung und von Tomaschny für die Harnsäureausscheidung gefundenen korrespondieren, identifiziert werden, wobei die Vormittagserhebung unabhängig von der Nahrungsaufnahme, das Nachmittagsmaximum aber in Abhängigkeit von der Mittagsmahlzeit steht. Nach Witt kann es sich bei JKDarreichung nicht um eine Steigerung des Stoffwechsels handeln, sondern die diesbezügliche Mehrausscheidung ist auf eine erhöhte Tätigkeit der Niere, welche durch einen gesteigerten in das Blut erfolgten Übertritt der Stoffwechselprodukte hervorgerufen wird, zurückzuführen. Am Vormittag kann nicht die Nahrungsaufnahme das ausschlaggebende Moment sein, da auch Hungertage das ron 9-11 Uhr vormittags bestehende Maximum ergeben, sondern die im wachen Zustande erböhte Funktion der Niere. Die Einflüsse, die den Menschen während dieses Zeitabschnittes treffen, entgegen der Abhaltung derselben während des Schlafes, stellen die maßgebenden Faktoren dar.

Für das Sajodin kann ich eine Entscheidung nicht fällen, da das Sajodin im Organismus abgebaut wird, daher nur ein Hungertag über das Vormittagsmaximum Aufschluß geben kann.

In noch frapanterer Weise als in den bisherigen Resultaten zeigt sich die Gleichmäßigkeit der Jodausscheidung im Tagesquantum zu Gunsten des Sajodins im Gegensatz zu JK in folgendem Versuch (Versuch III):

Eingeführt 8 Uhr früh

$12 \%$ mittag 8 abends je $1 \mathrm{~g}$ Sajodin.

$\%$ Verhältnis zur Gesamtausscheidung

Ausscheidung am 1. Tag $175.6 \mathrm{mg}$ Jod .... $5546 \%$

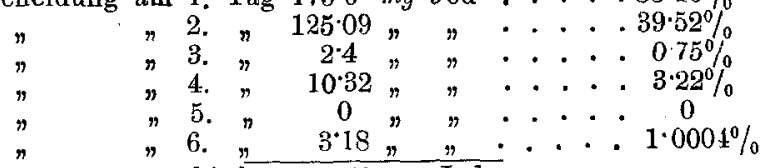

In Summa ausgeschieden $31659 \mathrm{mg}$ Jod. 
Die Jodtherapie in ihren Bezieh, z. quant. Jodausscheidung. 293

Dagegen zum Vergleich der IV. Versuch von Anten:

Eingeführt $7 \mathrm{Uhr}$ morgens

In Summa ausgeseh. $1289^{\circ} \mathrm{mg}$ JK.

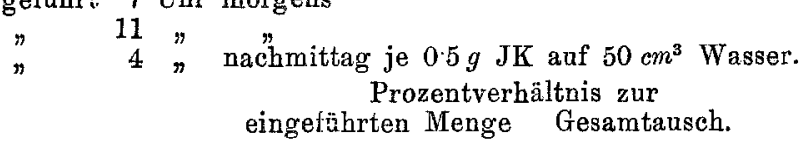

1. Tag $1084 \mathrm{mg}$ JK $7229 \%$. . . $84.06 \%$

$2 . " 189.3 \%, 12 \cdot 16 \%$. . . $1467 \%$

3. $" 15.8 \% 1 \cdot 03 \% \ldots . .1 \cdot 22 \%$

4. " $0.4 \% " 0.03 \% \ldots . . .0 .33 \%$

Wohl ist der Unterschied in den Darreichungszeiten zu berïcksichtigen. A n ten verabfolgt die letzte Dosis in der 9., ich in der 12. Stunde. Die kollosale Differenz von $70 \%$ in der Jodausscheidung nach JK zwischen dem ersten und zweiten Tag kann aber nicht durch die um 3 Stunden früher erfolgte Darreichung erklärt werden.

Wenn die letzte Dosis Antens auch um $7 \mathrm{Uhr}$ abends gegeben worden wäre, so hätte das Maximum der Ausscheidung noch in die Nacht fallen müssen, so daß zugunsten des zweiten Tages nur ein kleines Plus sich ergeben hätte.

Beim Sajodinversuch prägt sich aber die gleichmäßige Ausscheidung in einer geringen Prozentspannung von $16 \%$ zwischen erstem und zweitem Tag aus.

Meine Versuche sind an jugendlichen und nierengesunden Individuen angestellt, was mit Rücksicht auf die Mitteilung von $\mathrm{Chelmowski}$ und Hildebrand, die nach Applikation von JK bei älteren Leuten eine verlängerte Jodausscheidung finden (quantitative Versuche sind nicht angestellt) ausdrücklich hervorgehoben sei. Ich habe nun einem Nephritiker, 25jähriger Lupus faciei mit Eiweißausscheidung und spärlichen hyalinen '/ylindern im Sediment $2 \mathrm{~g} 10 \%$ Jodipin um 9 Uhr früh verabreicht. Die Ausscheidungszahlen sind folgende:

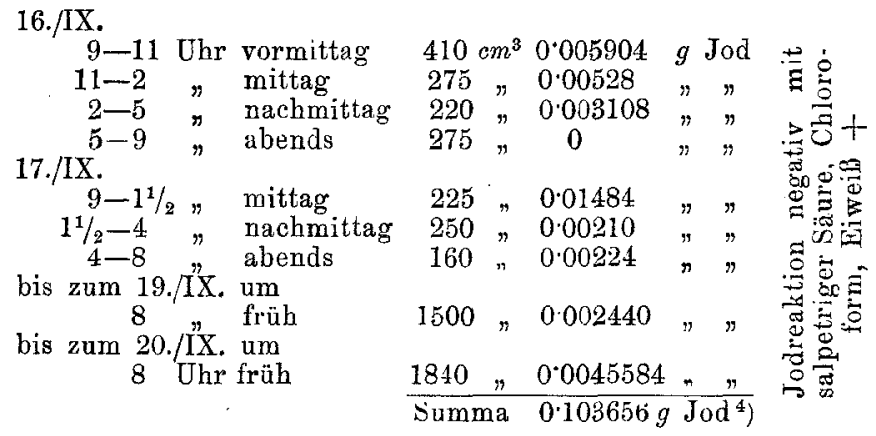

1) Der Versuch wurde abgebrochen, da nach 4 Tagen die Beendigung der Ausscheidung erwartet worden war. 
Es wird also innerbalb 5 Tagen $0 * 103656$ Jod ausgeschieden. Dieses Quantum (zirka 50\% der eingeführten Menge) ist als Minimum genügend, um zu sagen, $\mathrm{da}$ h dem Normalen gegenüber keine Retention aufgetreten ist, im Vergleich zu den Singerschen Zahlen, denen zufolge das Ausscheidungsmittel $58.5 \%$ Jod bei einer Schwankungsbreite von $49 \cdot 2-69 \%$ beträgt. Bemerkenswert ist es, daß wir Jod mit der salpetrigen Säure-Chloroformprobe nicht nachweisen konnten. Auch sei die Tatsache, daß das Maximum der Jodausscheidung auf den 4. Tag fällt, ein Umstand, der mit den Resorptionsverhältnissen bei Nierenkranken zusammenhängen lann, gebührend hervorgehoben. Bisher verdanken wir bloß Jennys Arbeit Daten über JK-Ausscheidung bei Nephritis mit quantitativen Belegen. Wir sehen im Versuch II. Jennys das Maximum der Ausscheidung erst in die 4. Stunde fallen. Während in den Normalversuchen Antens (I. Versuch und II.) nach 12 Stunden schon mehr als die Hälfte der Gesamtausscheidung erreicht war, ist die Elimination bei chronischer Nephritis nur $35 \cdot 12 \%$, also weniger als die Hälfte der Gesamtausfuhr. Jodipin hat nun in meinem Versuch noch größere Differenzen gezeigt. Die Beziehungen zwischen JKAusscheidung und Nephritis werden in einer demnächst erscheinenden Arbeit von Dr. Sobotka und mir zur Erörterung gelangen.

\section{Retention des Jods.}

Bei Betrachtung der Ausscheidungsresultate des Sajodinversuchs ergibt sich die Frage, wo denn eigentlich der Rest dẹs nicht zur Ausscheidung gelangenden Jods im Organismus verbleibt.

Als krasses Beispiel sei der Versuch von Lifschitz angeführt, in welchem von $343 \mathrm{~g}$ JK nur 244.738 ausgeschieden wurden, wo also ca. $100 \mathrm{~g}$ im Organismus zurückgehalten blieben, obwohl die Untersuchung noch 12 Tage nach dem Aufbören der JK Therapie fortgesetzt wurde, aus äußeren Gründen nicht bis zur Jodfreiheit des Urins. Dieser weist in den letzten Tagen allerdings nur $m g$ von Jod auf. 
Die Jodtherapie in ihren Bezieh. z. quant. Jodausscheidung. 295

Lasse ich die Frage des physiologischen Jodgehaltes der Zelle unberücksichtigt, so ergibt sich, da. die Speicheldrüse als Exkretionsorgan nicht in Betracht kommt, da der Speichel geschluckt wird. Die im Nasenschleim enthaltenen Mengen sind sehr gering $0.9-1.5 \%$ der eingeführten Menge. Doch haben die Zahlen A n tens nur für das beim Jodschnupfen sezernierte Sekret Geltung und können auf das normale Nasensekret nicht übertragen werden.

Die in Fäces ausgeschiedenen Mengen sind außerordentlich gering.

Lifschitz fand in diarrhoischen Stühlen nach Darreichung von $36 \mathrm{gJK}$ an den Untersuchungstagen 0.045, 0.052 $g$ JK, Fricker beziffert die Ausscheidung durch die Galle mit $0.86 \%$ (nach $1 \mathrm{~g}$ Lithionjodat per os gereicht) und Benedicenti mit $0.05 \%$ durch das Pankreassekret. How al d fand nach Darreichung von $1-3 \mathrm{~g} \mathrm{JK}$ pro die in $10 \mathrm{~g}$ Haaren $0.1 \mathrm{mg} \mathrm{J}$ im Fettextrakt, $0.3 \mathrm{mg}$ im Haar selbst, in einem anderen Fall $1.8 \mathrm{mg}$ im Fettextrakt und $0.8 \mathrm{mg} \mathrm{J}$ im Haar selbst. Der Wasserextrakt war jodfrei. Nach K ellermann wurden nur geringe Mengen im Schweiße entleert, 0.00035 in $70 \mathrm{~cm}$ Schweiß bis $0.00138 \mathrm{gJK}$ in $115 \mathrm{~cm}$ Schweib, nach längerer täglicher Darreichung von $2 \frac{1}{2} g \mathrm{JK}$.

Über die perspiratio insensibilis (siehe $\mathrm{S} \mathrm{chw}$ e $\mathrm{nken-}$ bechers Versuche über die NaCl-Ausscheidung bei derselben) bestehen keine Angaben. Die Ausscheidung durch die Talgdrüsen muß erst bewiesen werden, da die Angaben ron A damkiewicz unzulänglich sind. Der Schilddrüse, die eine große Rolle im Jodstoffwechsel spielt, kommt eine entscheidende Rolle bei der Retention nicht zu.

Wir müssen also gestehen, daß wir über die Schicksale des im Körper retinierten Jods nach Jodkalidarreichung nicht orientiert sind. Auch die Befunde Löbs, der bei einem Hund nach langer Jodkaliumdarreichung 72 Stunden nach der Tötung im Blute und in der Leber, bei einem Hund 48 Stunden nach der Tötung nur in der Leber eine jodhältige, in Alkohol nicht übergehende, wahrscheinlich Jodeiweiß darstellende Verbindung fand, geben nach dieser Richtung nicht genügenden Aufschluß.

Während man bei der Einführung der Jodeiweißverbindungen von der Ansicht ausging, daß sie als solche 
zur Resorption gelangen ( $\mathrm{Z} \mathrm{u}$ e l z e r), haben die jüngsten Untersuchungen von $\mathrm{F} \ddot{\mathrm{u}} \mathrm{rth}$ und $\mathrm{Friedmann}$ ergeben: Im Darmsafte fand sich nach Darreichung von Jodalbazid während der Verdauung die Hauptmenge des Jods in Form anscheinend hochmolekularer, durch Phosphorwolframsäure fällbarer Verbindungen. Nach vollzogener Resorption ändert sich das Verhältnis, aber nicht erst im Blute, sondern bereits in der Darmwand, insoferne als sich die Hauptmenge in derselben in anorganischer Form findet. Die Resorption des Jodalbazid eriolgt also nicht in Form hoch molekularer Produkte, wie man sie nach Einwirkung von Verdauungsfermenten auf Jodeiweißkörper auftreten sieht. Diese Tatsache spricht entschieden gegen die Annahme Boruttaus, der nach Jodglidinedarreichung Jod in fester Bindung im Harn findet und sich in seinen Befunden auf $\mathrm{Hofmeister}$, Neuberg und Mosso stützt, die nach Zufuhr von Jodeiweiß Übertritt von solchem bzw. von Orthojodhipursäure im Harn nachgewiesen haben. Ganz abgesehen davon, daß die jodbindende Kraft des Harnes bei der Konstatierung gebundenen Jods nicht übersehen werden darf, ist die Heranziehung $\mathrm{H}$ of meisters unverständlich, da dieser erst nach intravenösen Injektionen großer Dosen (1 $g$ auf $1 \frac{1}{2}$ Kilo Kaninchen) alkalisch gemachten kristallisierten Eieralbumius Jodeiweiß in den Harn übergehen sah, nach geringerer Menge aber nur $\mathrm{J}_{0} \mathrm{dalkali}$ im Harne wieder fand. Die großen Dosen Neubergs und Mossos beim Kaninchen lassen eine Übertragung der Versuche auf den menschlichen Organismus nicht $\mathrm{zu}$.

Das Jothion (Ureser) durchdringt die Haut in Dampfform nicht, sondern wird seiner Löslichkeit in der Gewebelymphe entsprechend (bei $30^{\circ} \mathrm{C} 134^{\%} \%$ ) resorbiert.

Die Jodfette werden nach den trefflichen Untersuchungen von Winternitz im Speichel und Magensaft nicht angegriffen, und erst im Darm resorbiert, der Form nach aber so, daß bei der Fettspaltung das Jod ungemindert an die Fettsäure gebunden bleibt. Bei den üblichen therapeutischen Dosen von $15-20 \mathrm{~g}$ pro die wird das Jodipin fast vollständig oxydiert und nur ein kleiner Teil des resorbierten Jodipins als Organfett im Unterhautzellgewebe, Knochenmark, Leber und Herzmuskel deponiert. Für die Praxis 
Die Jodtherapie in ihreu Bezieh. z. quant. Jodausscheidung. 297

ist der Verlust durch den Kot ohne Bedeutung. Ein $50 \mathrm{~kg}$ wiegender Patient nahm innerhalb 10 Tagen $390 \mathrm{~g}$, pro die also fast $40 \mathrm{~g}$. Der Kotverlust betrug rund $4 \%$. Auch die bei der subkutanen Injektion in den Kreislauf gelangenden Mengen fallen zum größten Teil der Oxydation anheim, zum kleineren Teil der Depotbildung in den Organen. Bis zu einem gewissen Grade ermöglichen die Ernährungsbedingungen, Mast oder Hunger, dem Arzte eine Regulierung der Jodausscheidung. Die spaltende Wirkung der Blutalkaleszenz kommt ebenso wie bei der inneren Darreichung nur in geringerem Maße in Betracht.

Über die Wandlungen des Sajodins im Organismus ist nur bekannt, daß es als solches resorbiert wird; denn Magenpankreassaft und Steapsin bringen keine Zersetzung des Medikamentes hervor. Es muß mit großer Wahrscheinlichkeit eine Depotbildung dieser Kalkseife im Organismus angenommen werden.

\section{Beziehung der quantitativen Jodausscheidung zu den Neben- wirkungen.}

Ich wende mich nun der zweiten kardinalen Forderung zu, die wir an ein klinisch ideales Jodpräparat zu stellen haben. Ich versage es mir auf die Frage der Ursache des Jodismus einzugehen, bezüglich deren ich auf eine demnächst erscheinende Publikation von Dr. Sobotka und mir verweise. Vor allem möchte ich, bevor ich die Jodintoxikation einer Besprechung unterziehe, auf eine Mitteilung Boruttaus reflektieren, der dem Jodglidine Nebenwirkungen zuschreibt, wie wir es sonst an Schilddrüsenpräparaten zu sehen gewöhnt sind. Im ersten Falle, dem 0.15 pro die des Medikamentes gereicht wurden, tritt eine Steigerung der Stickstoffausscheidung bei sonst gleichbleibender Ernährung, welche mit der Jodglidinedarreichung einsetzt und aufhört, auf. Es ist nicht angegeben, ob diese Patientin und noch ein 2. männlicher Patient an einer Struma litten und so die Stickstoffmehrausscheidung auf Wirkungen zu setzen ist, wie sie nach kleinen JK-Dosen bei Kropf beobachtet werden (Thyreoidinwirkung). Jedenfalls bedürfte es genauerer Untersuchungen, bevor man zur Verallgemeinerung dieser Befunde schreitet. 
Die große Zahl der Präparate verdankt den Bestrebungen, den Jodismus zu vermeiden, ihr Entstehen. Die erste Bedingung für vergleichende Untersuchungen der Präparate ist nun der gleiche Gehalt an Jod in der dargereichten Dosis. So liest man, um ein geläufiges Beispiel anzuführen, oft in Publikationen, daß Sajodin in gleicher Dosis mit dem JK keinen Jodismus mache, wobei der Autor gar nicht bedenkt, daß $1 g$ Sajodin nur $0.24 \mathrm{~g}$ Jod, $1 \mathrm{~g}$ JK aber $0.765 \mathrm{~g}$ Jod enthält; also $3 \mathrm{mal}$ soviel als Sajodin. Als 2. Punkt ist zu berücksichtigen, wieviel der einverleibten Menge in Zirkulation gelangt, was man aus dem Harn, da die Niere das Hauptausscheidungsorgan des Jods ist, leicht berechnen kann, der 3. Punkt: wie verteilt sich die Menge des resorbieten Jods auf die Zeiteinheit, kann beim Studium dieser Frage nicht außer acht gelassen werden.

Am günstigsten dürfte theoretisch postuliert das Präparat wirken, welches bei langsamen Anstieg die kleinste eben wirksame Dosis Jod in längerer Dauer in der Zirkulation festbält und allmählich ausschleichen läßt. Dieser Forderung wird am ehesten die Jodipininjektion gerecht, denn erst am 10.-15. Tage wird $0.1 \mathrm{~g}$ Jod pro Liter ausgeschieden (siehe meine kritischen Bemerkungen zu den Zahlen Feibes, p. 287), dann hält die Jodausscheidung, wenn der Versuch von Winternitz am Hund auf den Menschen bezogen werden darf, gleichmäßig an und klingt allmählich $a b$.

Tatsächlich sind bisher meines Wissens auch keine Fälle von $\mathrm{schwerem}$ Jodismus während einer Jodipininjektionskur beschrieben worden. Soll dabei nur der Zufall seine Hand im Spiele haben? Ob aber das gleichmäßig langsame Ansteigen, auf das, so viel mir bekannt ist, noch nicht der gehörige Nachdruck gelegt wurde, nicht gerade auch bei Jodiosynkrasischen eine Gewöhnung an das Mittel bedingt und dadurch Vermeidung der schweren Jodismusformen, möchte ich zur Diskussion stellen."

Immerhin wird man gut tun, nebst Beobachtung der übrigen Kautelen (Eiweißfreiheit des Harns, Berücksichtigung der Konstitution etc.) sich durch entsprechend kleine JK-Dosen zu überzeugen, ab die Idiosynkrasie nicht eine sehr hochgradige ist, da nach Einführung einer größeren Menge, die Entfernung des Depots, wenn hochgradiger Jodismus aufgetreten ist, große Schwierigkeiten bereiten würde. 
In striktem Gegensatz dazu steht das JK, das nach innerlicher Einnahme innerhalb der ersten 2-3 Stunden den Organismus überschwemmt, wobei der Überschuß in kürzester Zeit den Körper wieder durch die Niere verläßt. Der rasche stürmische Anstieg scheint in Beziehung zum Jodismus zu stehen. Die Mitte zwischen diesen beiden Extremen halten von den übrigen J-Präparaten die Jodeiweißkörper und die Jodfette bei innerlicher Darreichung. Die quantitativ ausgeschiedenen JMengen während des Anstieges, der Dauer der Elimination, die Verteilung auf die Zeiteinheit variieren mehr oder minder, wie aus den obigen Zahlenangaben zu ersehen ist.

Nur dem Sajodin, dem jetzt aktuellsten Präparat, seien einige Worte gewidmet. Sehen wir im Falle II die Gesamtausscheidungsresultate an, so ergibt sich, dab von $1 \mathrm{~g}$ Sajodin $0.07205 \mathrm{~J}=0.0942 \mathrm{JK}$ ausgeschieden werden. Da die JK-Ausscheidung im Harne $75 \%$ beträ!t, so entspräche dieses nur einer JK-Einfukr von $0.125 \mathrm{~g}$. Im allgemeinen heißt es nun, daß $1 \mathrm{~g}$ Sajodin $1 / 3 \mathrm{~g} \mathrm{JK}$ entspricht, dem eingeführten Jodwert nach. Dem Ausscheidungswert nach entspricht es aber nur $1 / 8 g$ JK. Auf die häufig verschriebene Lösung von $5 g \mathrm{JK}$ auf $200 \mathrm{~g}$ berechnet, ergibt sich, daß einem Eßlöffel dieser Solution (das ist $15 \mathrm{~g}$ ) der 13 . Teil derselben $0.383 \mathrm{JK}$ entspricht. $0.125 \mathrm{JK}$, der Jodausscheidungswert des Sajodins, entspräche dann dem dritten Teile eines Eßlöffels i. e. einem Kaffeelöffel $(5-6 \mathrm{~g})$ gerechnet. Es würde also dieser Rechnung nach ein Kaffeelöffel einer Lösung von $5 \mathrm{zu} 200 \mathrm{JK} 1 \mathrm{~g}$ Sajodin (also zwei halbgrammigen Pastillen) in seiner Jodwirkung entsprechen.

Sehen wir nun Fall 3 nach dieser Richtung durch. Von $3 g$ Sajodin entsprechend $1 \mathrm{~g} \mathrm{JK}$ werden ausgeschieden $0.3166 \mathrm{~g}$ $\mathrm{J}=0.414 \mathrm{JK}$. In derselben Weise, wie oben berechnet, ergibt dies eine JK-Einfuhr von 0.552 , also nur die Hälfte der eingeführten JK-Menge vom Standpunkte des Ausscheidungswertes angesehen. Es würden also $3 g$ Sajodin durch $1 \frac{1}{2}$ Eßlöffel einer $5 \%$ JK-Lösung ersetzt; daraus folgt, was schon für die Jodeiweißpräparate von Japha in der Diskussion zum Lesserschen Vortrag betont wird, daß das relativ seltene Auftreten von Jodismus in erster Linie der kleinen J-Menge, die in Zirkulation gerät, zugeschrieben werden muß. Aber geradeso wie 
bei der Jodipineinspritzung der allmähliche Anstieg, der bei derselben allerdings auf 10-15 Tage verteilt ist, als Grund für eine Gewöhnung angeführt wurde, geradeso scheint mir hier, das allerdings nur Stunden betreffende Einschleichen des $J$ und die gleichmäßige Verteilung des $J$ in der Zeiteinheit, von Bedeutung für die Tatsache des seltenen Auftretens des Jodismus nach Sajodindarreichung. Selbstrerständlich wird aber (Sitvenia verbo) bei absoluter Jodidiosynkrasie Jodismus nicht vermieden werden können und ich verweise nur auf die beiden schweren Fälle von Jodintoxikation nach Sajodingebrauch, über die Eschbaum und Roscher berichtet haben.

Ich erinnere an eine Arbeit von Gundurow, der das anerkennenswerte Streben, sich Klarheit über die Wirkungsweise der Präparate zu verschaffen, in einem Falle von Jodismus der Thyroidea betätigte, und Jodkali-Rektalsuppositorien, Sajodin und Jodipin bei innerlichem Gebrauche miteinander verglich. Leider sind die quantitativen Verhältnisse der Ausscheidung und ihre oben erörterten Beziehungen zur Einfuhr unberücksichtigt geblieben, so daß ein bestimmter Schluß nicht gezogen werden kann. Daß aber alle drei Präparate Schwellung der Thyrioidea machten, bestätigt nur das zu erwartende Resultat.

Aber nicht nur die verschiedensten Arzneimittel wurden ersonnen, um den Jodismus zu vermeiden, auch ihre Anwendungsweise wurde mannigfach variiert. Lesser empfiehlt die Dosis refracta und die Darreichung von $J K$ in schleimigen Vehikeln, v. Zeissel die Anwendung von Klysmen (2 $g$ Jodnatrium oder Jodlithium mit 5 guttae Tinctura Opii), deren quantitative Ausscheidung noch nicht studiert ist. Die Tatsache, daß nur in einem geringeren Prozentsatz der so behandelten Fälle Jodismus entsteht, dürfte zum Teil wenigstens auf der geringen \% ischen Ausnützung der dargereichten Jodmenge beruhen. Die perkutane Anwendung des Jothion und der JK-Salben in ihren Prozentausscheidungsverhältnissen wurde bereits besprochen.

Der Vollständigkeit halber sei die Einführung von JK durch die Kataphorese (Karfunkel) erwähnt, doch sind die resorbierten Mengen sehr gering. In den Versuchen $5 \%$ JKLösung durch gesunde Haut zu kataphorisieren, zeigten sich 0.012727 $\mathrm{g} \mathrm{J}$ im Mittel im Harn, allerdings nur bei der Durch- 
Die Jodtherapie in ihren Bezieh. z. quant. Jodausscheidung. 301

leitung eines Stroms von 5 Milliampère durch $3 / 4$ Stunden hindurch. Bezüglich des subkutanen Weges sei auf die obigen ausführlichen Darlegungen verwiesen.

Es erübrigt noch zusammenfassend auf Grund der gewonnenen Daten die Ivdikationen zu erörtern, die sich für die Anwendung der einzelnen Mittel ergeben. Ist es notwendig rasch zu wirken, dann wird man zum JK greifen. Denn wir besitzen kein anderes Mittel, um mit diesem in so kurzer Zeit das Maximum der zirkulierenden Menge zu erreichen. Haben wir es mit blanden Krankheitsformen zu tun, so können wir die Jodeiweißpräparate und die Jodfette innerlich verwenden, insbesondere wenn JK nicht vertragen wird, doch in Erkenntnis des Umstandes, daß wir hier mit sehr viel kleineren Dosen operieren. Leider existieren über die Jodeiweilpräparate mit Ausnahme ron Jodglidine und Jodolen noch keine quantitativen Untersuchungen. Von den Jodfetten ist das Sajodin geschmacklos, während dem Jodipin ein unangenehmer, allerdings durch Korrigentien bis zu einem gewissen Grade behebbarer Geschmack zukommt, Momente, die bei der Wahl des Präparates in Betracht kommen. Will man den Patienten unter längere Jodwirkung setzen, ohne dah eine dringliche Indikation besteht, so kann man zur subkutanen Jodipininjektion greifen, der Tatsache sich bewußt, daß erst nach einer Woche eine nennenswerte Jodmenge zur Wirkung kommt. Ist die innerliche Anwendung erschwert (Benommenheit des Patienten, Widerwillen gegen Medikamente) oder ein Wechsel des Jodpräparates erwünscht, besteht Furcht vor der Injektion, so kommt die perkutane Jodaufnahme in Betracht, das Jothion an erster Stelle. Man darf jedoch nicht vergessen, daß es nach meiner Erfahrung und in Übereinstimmung mit Lifschitz an den mit undurchlässigem Verbandstoff bedeckten Körperstellen Hautreizungen hervorrufen kann. Über Jodvasogen-Schmierkuren (L e is tik ow) fehlt mir jede Erfahrung. Der teuere Preis der neuen Präparate wird bei der Indikationsstellung auch in die Wagschale fallen.

Zum Schluß sei eines Vergleichs gedacht, der sich einem beim Studium der früber auseinander gesetzten Verbältnisse aufdrängt.

Trotzdem bei Hg-Schmierkuren ungleich geringere Mengen von Quecksilber als nach der Injektionstherapie ausgeschieden 
werden, muß der Schmierkur unbedingte Heilkraft zuerkannt werden. Auch hier wird der allmählich steigenden Resorption und in der gleichmäBigen Zirkulation des $\mathrm{Hg}$ entgegen der Ửberflutung durch Hg-Injektionen und der raschen Ausscheidung des Quecksilbers bei denselben (Winternitz) ein Teil der Wirkung zuzuschreiben sein.

Wie Pilze nach einem Regen schießen neue Jodpräparate auf und man weil nicht, was man mehr bewundern soll, das Sprachtalent ihres Erzeugers oder seine chemische Erfindungsgabe. Da ist das Jodomenin, das Jodival und das Griserin, welch letzteres das $\mathrm{J}$ in Benzolkerne gebunden enthält. Noch kurz vor Abschluß dieser Arbeit das Allkuenta, eine wasserlösliche, jodbältige Alkoholsalbe.

Wünschenswert wäre es, daß man sich ror der klinischen Einführung über die quantitativen Ausscheidungsverhältnisse des Jods und das physiologische Verhalten der Präparate im Organismus orientiere. Wer wird sich aber dieser vielleicht fruchtlosen und jedesfalls mühevollen Arbeit unterziehen?

Zum Schlusse ist es mir eine angenehme Pflicht Herrn Prof. Kreibich, Vorstand der dermatologischen Klinik, und Herrn Prof. v. Zeynek, Vorstand des Institutes für physiologische Chemie und seinem Assistenten Herrn Dr. Lippich für die Überlassung des Materials, bzw. die freundliche Unterstützung meinen besten Dank zu sagen.

\section{Literatur.}

Abderbalden und Ka utzsch. Vergl. Unters. über die Ausseheidung von J. bei Verabreichung von JK u. Sajodin. Zeitschr. f. exp. Path. IV. Bd. 3. H. 1907. p. 315 .

A d a mkiewicz. Die Ansscheidungswege des Jodkaliums beim Menschen. Charité-Ann. III. p. 381. 1878; ref. nach Arch. f. Derm. u. Syph. Bd. XI. p. 114. 1879.

A nten. Über den Verlauf der Ausscheidung des JK im menschl. Harn. Arch. f. exp. Path. u. Pharm. Bd. XLVIII. p. 331. $190 \%$.

Bïnz. Die Zerlegung des JK im Organismus. Vireh. Arch. LXII. Bd. 1874. p. 124

- Beiträge zur pharmak. Kenntnis der Halogene. Archiv f. exper.

Path. XXXIV. Bd. 1894. p. 185.

Benedicenti. Zit. nach Fricke.

Boruttan. Über das Verhalten der Jodglidine im menschlichen $u$. Tierkörper. Deutsche med. Wochenschr. XXXIIII. Jahrg. 1907. p. 1490.

III. Bd. 1874 .

Buchbeim. Über die Wirkung des JK. Arch. f. exp. Pathol. etc. 
Die Jodtherapie in ihren Bezieh. z. quant. Jodausscheidung. 303

Busch und Gumpert. Jodomenin, ein neues int. Jodpräparat. Therapie der Gegenw. 1908. April. Zit. nach Zentralbl. für ges, Medizin. 1908. Nr. 37.

Capeilmann. Unveröffentl. Unters. Zit. nach Heffter. Ergeb. d. Phys. II. Bd. p. 507.

Chelmowski. Über die Ausscheidung von J. und Salicylsäure in Krankheiten. Zit. nach Jahrb. f. Tierchemie. XXIV. 1894. p. 297.

Drese r. Zur Anwendung des Jothions. Berl. kl. Woch. 1905. Nr. 23.

Fs ch ba u m. Notiz über das Sajodin. Med. Kl. 1906. Nr. 18. p. 466.

F eibes. Betrachtungen über das Jodipin. Derm. Zeitschr. IX. Bd.

1902. (Sep.-Âbdruck.)

Feigl Exper. Unters. über den Einfluß von Arzneimitteln auf die

Magensaftsekretion. Biochem. Zeitschr. VIII. 1901.

$\mathrm{F}$ is chel. Klinische Beobacbtungen über den Heilwert des Jodipins.

Arch. f. Dermat, etc. LIII. Bd. 1. Heft. 1900.

Fischer und Mering, Über eine neue Klasse von jodhaltigen

Mitteln. Med. Klinik. 1906. II. Bd. Nr. 7. p. 157.

Fricker. Ausscheidung des Lithionjodats durch die menschliche

Galle. Biochem. Zeitschr. Bd. XIV. p. 286.

O. v. Fürth und M. Fri e d mann. Über die Resorptionsweise jodierter

Eiweißkörper. Arch. f. exp. Path. u. Ph. Festschr. f. S chm ie de ber g. 1908.

Gondurov. Beitrag zur Frage der Thyreoiditis acuta. Archiv für

Derm. u. Syph. 1906. Bd. LXXXIX. p. 399.

Heffter. Die Ausscheidung körperfremder Substanzen im Harn.

Ergeb. d. Phys. II. Jahrg. I. A bt. 1903. IV. Jahrg. 1905. p. 296.

- Über Antens Methode der quant. Jodbest. im Urin. Z. f. exp.

Path. II. Bd. 1906. p. 432.

Hill ebrand, Beitrag zur JK-Therapie der Psoriasis. Arch. f. Derm.

1892. Bd. XXIV. p. 931.

Hofmeister. Z. f. phys. Chemie. XXIV. 1898. p. 171.

Howald. Vorkommen und Nachweis von J, in den Haaren. Z. $f$.

phys. Chem. XXIII. Bd. $219-225$ oder Inaug.-Diss. Bern. 1897.

Jenny. Über die JK-Ausscheidung durch Diuretica nebst Unters.

über die Aussch. bei Nephritikern. Inaug.-Diss. Bern. 1904.

Karfunkel. Beitrag zur Kotaphorese. A. f. Derm. u. S. Bd. XLI. 1897. p. 10 .

Kämmerer. Über die arzneiliche Wirkungsweise d. JK u. Subli-

mats. Virchows Arch. LIX. Bd. p. 459.

- (Polemik gegen B in z.) Virchows Archiv. LX. Bd. p. 526. 1874.

Kellermann. Über die Ausscheidung von Jod im SchweiB. Z. f.

exp. Path. u. Th. 1905 . Nr. 1. p. 189 u. 687.

- Über die perkutane Resorbierbarkeit von Jod. Zeitschr. f. exp.

Path. u. Th. Bd. II. 1906. p. 416.

Krause. Über quant. Jodbestimmung im Harn. Ibidem. Band III. 1906. p. 365 .

Leistkow. Über Jcdvasogen als Ersatz für den internen Gebrauch

der Jodsalze. Monatsh. f. pr. Derm. XXIII. p. 625.

Lesser. Über das Verhaiten der Joäpräparate spez. des JK und

Jodipins im Organismus. Arch. f. Derm. u. Syph. LXIV. Bd. 1903. p. 91.

Lesser. Zur Kenntnis und Verbreitung des Jodismus. Deutsche

med. Woch. 1903. p. 849. Nr. 46. Diskussion zum Vortrage. Ibidem. 1903.

Nr. 46. p. 358 .

Lifschitz. Über Jodausscheidung nach gr. JK-Dosen etc. Archiv

f. Derm. Bd. LXXV. 1905. p. 354.

$\mathrm{L}$ i o $\mathrm{n}$. Die Resorption der Haut für JK in versch. Salbengrundlagen.

Festschrift für Kaposi. 1900. Ergänzungsband des Arch, f. Derm.

Löb. Die Jodverteilung nach Einfuhr versch. Jodverb. Arch. f. exp.

Path, etc. LVI. Bd. 1907. p. 320 . 
Marung. Über das Verhalten des J. zum Harn. Arch. int. de Ph. et Thér. (Separatabdruck.) 1900. p. 369.

Mosso und Neuberg. Über den phys. Abbau von Jodalbuminaten. Zeitschr. f. phys. Chemie. XXIV. p. 171. 1898.

Pi ck, F. J. Über die ther. Verwendung der Jodols. Arch. f. Derm. XVIII. Bd. 1886 . p. 583.

Peters. Über Jodipinresorption. Arch. intern. de Pharm. et Thér. Bd. XV. 1905. p. 189.

Polli o und Hirs chfeld. Über Resorption von Jod- u. JK-Salben.

Arch. f. Derm. u. Syph. LXXII. Bd. 1904. p. 163.

Roscher. Prakt. Erfahrungen mit Sajodin. Medizin. Klinik. 1906. Nr. 7. p. 164 .

Rose mann. Über den Verlauf der N-Ausscheidung beim Menschen. Pflügers Arch. Bd. LXV. 1897. p. 343.

Roeske. Zit. nach $W$ itt.

Röse l. Jodbestimmung im Harn und über Resorption von Jodolen etc. Pfiügers Arch. LXXVII. Bd. 1899. p. 22.

Scharlau. Kaspers Wochenschrift f. d. gesamte Heilkunde. 1842. p. 433 . Nr. 27 .

Schad e. Über d. Metall- u. Jodionen-Katalyse. Zeitschr. f. exper. Path. Bd. I. p. 603 .

Schierh of. Zur Pharmakologie der Jodverbindungen. Arch. intern. de Pharm. XIV. Bd. p. 429. 1905.

$\mathrm{Singer}$. Unters. über Jodausscheidung nach Gebrauch von JK und Jodipin. Zeitschr. f. klin. Med. Bd. LII. p. 521.

So $\mathrm{m}$ m e r f el d t. Über ein neues Jodeiweißpräparat Jodolen (Laqueur) etc. Arch. f. Derm. etc. LII. Bd. p. 29.

Tomaschny. Zit. nach Witt.

Walko. Über Jodbindungsvermögen des Harns. Zeitschrift für Heilkunde. XXI. Bd. 1900. (Sep.-Abdruck.)

Wesen berg. Die perkutane Jodapplikation. Therap. Monatshefte. 1905. XIX. Jahrg. p. 198.

Bd. LXXV. 1905 .

- Jothion, ein perkutan angew. Jodpräparat. Arch. f. Derm. etc.

- Zur Methodik der Jodbestimmung im Harn etc. Zeitschr. f. exp. Path. Bd. III. 1906. p. 367 .

Weland er. Über JK, JNa, Jodalbacid und Jodipin. Arch. f. Derm. u. Syph. LVII. Bd. 1901..p. 62.

W intern itz, H. Über die phys. Grundlagen der Jodipinther. Münch. med. Wochenschr. 50. Jahrg. 1903. p. 1241.

Winternitz, Rudolf. Über die Ausscheidung des $\mathrm{Hg}$ bei versch. Arten seiner Anwendung. Arch. f. D. u. Syph. 1889. Bd. XXI. (S.-Abdr.) Witt. Über den Verlauf der Jodausscheidung bei den Menschen. Inaug.-Diss. Greifswald 1905.

Xylander. Ü̉ber die Ausscheidung von Jod im Harn nach Appl. von Jodsalben. Inaug.-Diss. Würzburg. 1899.

v. Zeissl. Die Behandlung der Syphilis mit Jodklysmen. Ärztl. Praxis. 1905. Nr. 16. p. 181.

Zernik. Neue Arzneimittel, Spezialitäten etc. Dentsche m. Woch. 1907. 24. Januar. p. 43.

$Z$ uelzer. Neue Vorschläge zur Jodtherapie bei Syphilis. Arch. f. Jerm. Bd. XLIV 1898. p. 421. Festschritt für F. J. P i ck.

Die Arbeit von Georg Bas ch: „Über das Verhalten des Sajodins im Organismus" konnte ich leider nicht mehr berücksichtigen, da ich erst zur Zeit der Korrektur auf sie anfmerksam gemacht, sie mir nicht mehr beschaffen konnte. 